\title{
Zwischen Leitlinien und mutmasslichem Willen
}

Ulrich Erlinger

Korrespondenz:

Dr. med. Ulrich Erlinger, MPH

Stadtärztlicher Dienst

Walchestrasse 31

CH-8021 Zürich

ulrich.erlinger@zuerich.ch

\section{Einleitung}

Wenn bei demenzkranken Menschen im Pflegeheim Angst und Schmerz sichtbar werden, entspricht eine medizinische Behandlung dieser Symptome wohl praktisch immer dem mutmasslichen Willen der Betroffenen. Ob viele andere kurative und palliative medizinische Untersuchungen und Behandlungen an demenzkranken Menschen im Heim deren mutmasslichem Willen entsprechen, ist weniger eindeutig. Bei einem Verlust der Urteilsfähigkeit der Betroffenen müssen die Entscheidungen zudem vertretend gefällt werden [1-3], was den Entscheidungsprozess erschwert

Behandlungen wie zum Beispiel eine orale Antikoagulation (OAK), eine Substitution mit Vitaminen oder eine leitlinienkonforme im Vergleich zu einer gelockerten Einstellung eines Diabetes mellitus fördern kurzfristig die Lebensqualität der Betroffenen kaum. Zwar verhindern diese Behandlungen mittelfristig auch Krankheiten und Zustände, die die Lebensqualität zusätzlich beeinträchtigen. Sie wirken aber auch potentiell lebensverlängernd und entsprechen aus diesem Grund möglicherweise nicht dem mutmasslichen Willen der von stationärer Pflege abhängigen Menschen.

\section{Indikation, Aufklärung und Einwilligung} bei demenzkranken Menschen im Pflegeheim Die Entscheidung über den Beginn einer medizinischen Behandlung, aber auch über deren Fortsetzung, basiert auf deren Wirkung, möglichen Nebenwirkungen und Kontraindikationen, also letztlich auf einer Risiko-Nutzen-Abwägung und einer Kosten-Nutzen-Abwägung mit Rücksicht auf den individuellen Lebenskontext des Patienten. Eine Demenz, hohes Alter und Multimorbidität erschweren die Mitarbeit der Patienten bei der Behandlung und führen zu einer Vergrösserung ihrer Risiken [4-7].

Für Menschen mit fortgeschrittenen Demenzerkrankungen ist ein Verständnis der Vor- und Nachteile zum Beispiel einer OAK als Voraussetzung für eine rechtsgültige Aufklärung nicht möglich. Sie sind bezüglich dieser Entscheidung nicht urteilsfähig. An ihrer Stelle entscheiden Bezugspersonen und die behandelnden Ärzte. Eine Risiko-Nutzen-Abwägung zum Beispiel in Bezug auf eine OAK ist selbst für gesunde medizinische Laien nicht immer einfach. Besonders bei der Behandlung chronischer Krankheiten vor dem Hintergrund einer vorliegenden Demenz mit stationärer Pflegebedürftigkeit und den potentiellen Auswirkun-

\section{Entre directives et volonté présumée - Traitements médicaux préventifs des personnes atteintes de démence avancée dans les homes médicalisés}

De nombreuses personnes atteintes de démence sont, en raison de maladies internistes, soignées au moyen d'une médication préventive contribuant à les maintenir à domicile. Mais l'évolution de la pathologie démentielle les amène très souvent dans un foyer médicalisé suite à la perte d'autonomie. Elles continuent à y recevoir les traitements initiaux alors qu'elles sont devenues incapables de discernement. La plupart du temps, on ne sait pas vraiment si la poursuite d'un traitement préventif correspond à leur volonté présumée, compte tenu des conditions de vie différentes des précédentes. Les auteurs de l'article présentent les implications juridiques des traitements préventifs appliqués dans les homes médicalisés à des personnes atteintes de démence et incapables de discernement. Ils recommandent notamment de prendre plus fortement en compte la volonté présumée de ces personnes quant à leur vie en foyer médicalisé sur la base des valeurs générales de la société.

gen auf die Lebensqualität und die Lebensdauer im Pflegeheim sind Bezugspersonen stark gefordert, wenn sie den mutmasslichen Willen der Betroffenen abschätzen wollen.

Nach der Indikationsstellung und der Aufklärung ist die Einwilligung eine weitere Voraussetzung einer nicht notfallmässigen medizinischen Behandlung. Die Beweislast für das Vorliegen einer eindeutigen Einwilligung zur Behandlung liegt beim Arzt. Er muss im Streitfall beweisen, dass die Aufklärung erfolgt ist und der Patient in die Behandlung eingewilligt hat [8]. Der bezüglich einer komplexen medizinischen Ent- 
scheidung aufgrund der fortgeschrittenen Demenzerkrankung nicht mehr urteilsfähige Mensch kann keine rechtsgültige Einwilligung zur Behandlung geben. Der Arzt muss deshalb versuchen, den mutmasslichen Willen des Patienten herauszufinden. Dabei können Angaben von Angehörigen helfen, möglicherweise die Art und Weise, wie der Patient vor der Demenzerkrankung mit seiner Gesundheit umgegangen ist, und gegebenenfalls Ausführungen in einer Patientenverfügung $(\mathrm{PV})[2,3,9,10]$. handlung des Einzelnen allerdings keinen Stellenwert haben, weil sie keinesfalls der individuellen Meinung des Betroffenen entsprechen muss [9]. Sie kann nur der Plausibilisierung einer bekannten ablehnenden Haltung des Betroffenen dienen.

Lebensqualität von Menschen mit Demenzerkrankungen lässt sich differenziert in verschiedenen Dimensionen messen, die sich auf den Moment beziehen. Ein Kohärenzgefühl zum Beispiel, das sich auch auf eine über den Moment hinausgehende Lebenszeit

\section{Nicht immer ist klar, ob die Fortsetzung einer vorbeugenden Behandlung vor dem Hintergrund der veränderten Lebenssituation dem mutmasslichen Willen der Betroffenen entspricht}

Bei vielen betroffenen Menschen ist eine ablehnende Haltung gegenüber einem Pflegeheimaufenthalt bekannt. Eine grösstmögliche Lebensqualität bis zum Lebensende war fast allen Menschen auch in gesunden Zeiten wichtig [9]. Die möglicherweise in gesunden Tagen geäusserte Haltung des Betroffenen bezüglich eines Heimaufenthaltes ist von den für ihn entscheidenden Personen als Wertanamnese zu erheben [11]. Die ablehnende Haltung der überwiegenden Mehrheit der Menschen gegenüber einem Leben im Pflegeheim kann als allgemeine Werthaltung unserer Gesellschaft gesehen werden [12-14]. Vor dem Hintergrund dieser allgemeinen Werthaltung ist eine vorbekannte ablehnende Haltung eines Betroffenen als plausibel zu werten, auch wenn sie nicht schriftlich niedergelegt wurde. Für sich allein genommen darf die allgemeine Werthaltung in der Gesellschaft für die Be-

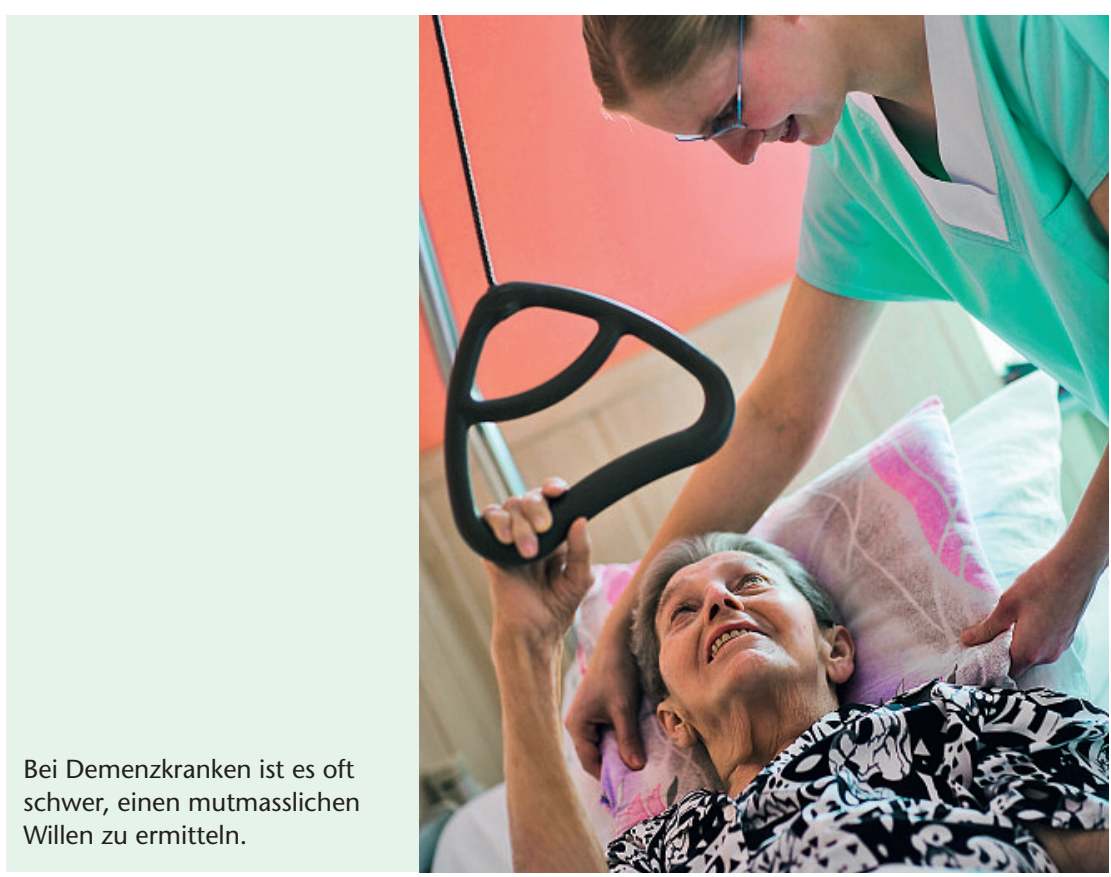

bezieht, gehört nicht dazu $[15,16]$. Eine sichtbar gute Lebensqualität von fortgeschritten demenzkranken Menschen ist demnach dem momentanen Wohlbefinden zu verdanken. Dieses Wohlbefinden bedeutet aber nicht, dass der demenzkranke Mensch seine vorbekannte ablehnende Einstellung bezüglich lebensverlängernder Massnahmen im Pflegeheim geändert hat. Dieses Wohlsein, im Moment isoliert betrachtet, stellt im Zusammenhang mit einer bekannten ablehnenden Haltung gegenüber lebensverlängernden Massnahmen im Pflegeheim auch vor dem Hintergrund der oben beschriebenen allgemeinen Werthaltung in der Gesellschaft keine Einwilligung in lebensverlängernde Behandlungen dar. In diesem Wohlsein in Würde zeigt sich primär die aktuell gute Lebensqualität, die sich der Patient auch in früheren Zeiten gewünscht hat. Diesem Wohlsein im Moment und dem Erhalt der Menschenwürde trotz Demenz gilt unsere FürsorgeVerpflichtung, der wir mit guter Betreuung gerecht werden müssen [9]. Gute Lebensqualität und der Verzicht auf medizinische Lebensverlängerung sind zwei Anliegen, die bei fortgeschritten demenzkranken Menschen im Pflegeheim durchaus zusammengehören können. Bei vielen Ärzten ist jedoch die Sorge verbreitet, mit dem Vorwurf konfrontiert zu werden, nicht gemäss den Regeln der ärztlichen Kunst gehandelt zu haben, wenn trotz fehlender mutmasslicher Einwilligung auf eine vorbeugende Behandlung verzichtet wird [3]. In jedem Fall ist es das erste Ziel, einen Konsens aller Beteiligten über eine sinnvolle Behandlung herzustellen, die dem mutmasslichen Willen des Betroffenen entspricht $[10,17]$.

Aus den oben aufgeführten Erwägungen leiten sich folgende Empfehlungen ab:

- Zwar werden von Patientinnen und Patienten möglichst einfache PV bevorzugt [18], der Themenkomplex «Demenz, Pflegeheim und Behandlungsoptionen» ist aber für viele Menschen so wichtig, dass es eine PV-Version geben sollte, in der sie für ihre Zukunft dazu Stellung nehmen kön- 
nen. Dort sollte die Möglichkeit des Verzichts auf vorbeugende Behandlungen für den Fall explizit festgelegt werden können, dass der Betroffene an einer Demenz leidet, bezüglich seiner Behandlung dauerhaft urteilsunfähig ist, irreversibel auf stationäre Pflege angewiesen ist und die Behandlung die momentane Lebensqualität nicht fördert. In Entscheidungen über medizinische Behandlungen sollte auch ohne vorliegende PV eine in gesunden Zeiten geäusserte Ablehnung eines Lebens im Pflegeheim auch vor dem Hintergrund der allgemeinen diesbezüglichen Werthaltung in der Gesellschaft noch mehr beachtet werden.

\section{Dem Wohlsein im Moment und dem Erhalt der Menschenwürde trotz Demenz gilt unsere Fürsorgeverpflichtung}

2 Patientinnen- und Patientengesetz des Kantons Zürich. Kanton Zürich; 2004.

3 Fellmann W. Arzt und das Rechtsverhältnis zum Patienten. In: Arztrecht in der Praxis. Zürich: Schulthess; 1997. S. 103-231.

4 Bauer J, Sieber CC. Antikoagulation bei älteren Menschen. Schweiz Med Forum. 2004;(4):824-831.

5 Robert-Ebadi H, Gal GL, Righini M. Use of anticoagulants in elderly patients: practical recommendations. Clinical Interventions in Aging. 2009;(4):165-177.

6 Lacut K, Gal GL, Mottier D. Primary prevention of venous thromboembolism in elderly medical patients. Clinical Interventions in Aging. 2008;(3):399-411.

7 Tincani E, Crowther MA, Turrini F, Prisco D. Prevention and treatment of venous thromboembolism in the elderly patient. Clinical Interventions in Aging. 2007;(2)237-246.

8 Bergmann KO. Selbstbestimmungsaufklärung und Patienteneinwilligung. In: Die Arzthaftung. Berlin: Springer; 1999. S.65-111.

9 Rüegger H. Herausforderung Demenz - Ethische Aspekte im Umgang mit demenzkranken Menschen. Intercura.2009;(105):4-36.

10 SAMW. Recht der Patientinnen und Patienten auf Selbstbestimmung - Medizinisch-ethische Grundsätze der Schweizerischen Akademie der Medizinischen Wissenschaften SAMW. Basel: vista point; 2005.

11 Putz W, Steldinger B. Patientenrechte am Ende des Lebens, München: Deutscher Taschenbuch Verlag; 2007.

12 Fleischer S et al. Growing old at home - A randomized controlled trial to investigate the effectiveness and cost-effectiveness of preventive home visits to reduce nursing home admissions: study protocol (NCT00644826). BMC Public Health. 2008;(8)

13 Gaugler JE, Duval S, Anderson KA, Kane RL. Predicting nursing home admission in the U.S: a meta-analysis. BMC Geriatrics. 2007(7).

14 Deutscher Altenpflege-Monitor 2007/2008: Generation 50plus: Einstellungen zur Altenpflege. Was erwartet der Kunde von morgen? Hannover: Pressestelle Altenpflege-Monitor; 2007.

15 Drageset J et al. Sense of coherence as a resource in relation to health-related quality of life among mentally intact nursing home residents a questionnaire study. Health and Quality of Life Outcomes. 2008;(6).

16 Becker S, Kruse A, Schröder J, Seidl U. Das Heidelberger Instrument zur Erfassung von Lebensqualität bei Demenz (H.I.L.DE.) Dimensionen von Lebensqualität und deren Operationalisierung. Z Gerontol Geriat. 2005;(38):108-121.

17 Putz W, Steldinger B. Behandlung nach dem Willen des Patienten. In: Patientenrechte am Ende des Lebens. München: Deutscher Taschenbuch Verlag; 2007: S. 37-47.

18 Harringer W, Hoby G. Die «PAVE»-Praxisstudie Patientenverfügung in der hausärztlichen Praxis. Schweiz Ärztezeitung. 2009;90(8):303-4.

19 Schweizerisches Zivilgesetzbuch - Erwachsenenschutz, Personenrecht und Kindesrecht. Die Bundesversammlung der Schweizerischen Eidgenossenschaft: Bundesblatt Nr. 1. 6. Januar 2009; 2008. 